\title{
魔力尺寸硒化镉纳米晶的压力稳定性研究
}

\author{
姜红梅, 赵文雅, 付瑞净, 肖冠军 \\ (吉林大学 超硬材料国家重点实验室, 物理学院, 长春 130012)
}

摘 要: 在极端条件下应用的耐压材料必须具有极高的结构和性能稳定性。魔力尺寸纳米晶体具有良好的结构、超 小的粒子尺寸和精确的原子组成, 逐渐成为研究的热点。采用胶体化学方法合成了硒化镉 $(\mathrm{CdSe})$ 魔力尺寸纳米晶, 其第一激子吸收峰位于 $463 \mathrm{~nm}$ 处, 相应的光致发光光谱表现出窄的半高宽, 仅为 $13 \mathrm{~nm}$ 。进一步利用金刚石对顶 砧压机研究了所制备的 CdSe 魔力尺寸纳米晶在压力下的光学响应和稳定性。研究结果表明: 随着压力的增大, $\mathrm{CdSe}$ 魔力尺寸纳米晶的吸收和发光峰位保持不变, 这种压力下的稳定行为与传统纳米材料在外界压力下所表现的 敏感特性相反; 压力卸下后, 样品仍然保持 CdSe 魔力尺寸纳米晶的原始结构和形态。CdSe 魔力尺寸纳米晶在加压 过程中表现出的良好耐压性能，有助于极端压缩条件下的魔力尺寸纳米材料的研究。

关 键 词: 魔力尺寸; 硒化镉; 耐压性能; 金刚石对顶砧

中图分类号: TQ174 文献标志码: A

\section{Pressure-resistance of Magic-sized Cadmium Selenide Nanocrystals}

\author{
JIANG Hongmei, ZHAO Wenya, FU Ruijing, XIAO Guanjun \\ (State Key Laboratory of Superhard Materials, College of Physics, Jilin University, Changchun 130012, China)
}

\begin{abstract}
Exploration of pressure-resistant materials largely facilitates the operation under extreme conditions where the stable structure and properties are highly desirable. Magic-sized nanocrystals (MSNs) are of great interest due to their well-defined structure, ultra-small particle size, and precise atomic composition. Here, we reported the colloidal synthesis of CdSe MSNs with a sharp first exciton absorption peak at $463 \mathrm{~nm}$. The corresponding photoluminescence (PL) spectrum exhibited a very narrow full width at half-maximum (FWHM) of about $13 \mathrm{~nm}$. In situ high-pressure evolutions of PL and absorption unambiguously indicated that the peak position of as-prepared CdSe MSNs kept nearly unchanged with the pressure increasing. This behavior was completely different from conventional materials upon the stimulus of external pressure. Furthermore, the decompressed samples retained the original structure and morphology of CdSe MSNs. The properties of good pressure resistance of the CdSe MSNs are reflected in the process of pressurization, which improves the research of the magic-sized materials under extreme pressure.
\end{abstract}

Key words: magic size; cadmium selenide; pressure resistance; diamond anvil cell

收稿日期: 2020-07-08; 收到修改稿日期：2020-08-22; 网络出版日期：2020-10-19

基金项目: 国家自然科学基金(11774125); 吉林省科技发展计划(20190103044JH)

National Natural Science Foundation of China (11774125); Jilin Province Science and Technology Development Project $(20190103044 \mathrm{JH})$

作者简介：姜红梅(1996-), 女，硕士研究生. E-mail: jianghm18@mails.jlu.edu.cn JIANG Hongmei(1996-), female, Master candidate. E-mail: jianghm18@mails.jlu.edu.cn

通信作者：肖冠军，教授. E-mail: xguanjun@jlu.edu.cn XIAO Guanjun, professor. E-mail: xguanjun@jlu.edu.cn 
魔力尺寸纳米晶(Magic-Sized Nanocrystals, MSNs) 具有结构清晰、粒径小和原子组成精确等优点, 对 其研究可为深入理解和控制胶体纳米晶的成核和生 长过程提供新的视角。同时, 在合成过程中, 随着反 应周期的延长或温度的升高, MSNs 的含量不再增 加, 而结构却很稳定 ${ }^{[1-9]}$ 。MSNs 以其独特的物理和 化学性质而受到人们的广泛关注, 人们已成功制备 出了多种不同尺寸的 $\mathrm{MSNs}^{[3-7]}$ 。例如, GAO 等 ${ }^{[8]}$ 探 讨了胶体合金 MSNs 室温下的形成机制; YANG 等 ${ }^{[9]}$ 进一步研究了合金 MSNs 的化学合成、掺杂和转化 等等。 $\mathrm{YU}$ 等 ${ }^{[2-3]}$ 开发并利用一锅者方法合成了高质 量的硒化镉 $(\mathrm{CdSe})$ 半导体 MSNs, 样品尺寸离散单 一, 具有尖锐的吸收峰。CdSe MSNs 是决定纳米晶 由成核到生长的阶段产物, 其局域能量最小。与传 统的量子点相比, CdSe MSNs 呈现光吸收狭窄和峰 位稳定的特点, 这主要归因于精确的原子配比和尺 寸单一, 从而导致非均匀谱线的展宽很小 ${ }^{[10]}$ 。

$\mathrm{CdSe}$ 纳米晶具有窄带性和尺寸依赖性, 其光致 发光特性备受关注, 而光致发光强度和波长是评估 光电器件性能的两个关键参数。迄今为止, 人们采 用无机壳表面针化、低温抑制非辐射复合和引入阳 离子等方法, 在增强 $\mathrm{CdSe}$ 纳米晶的发光强度方面 取得了系列创新成果 ${ }^{[11-12]}$ 。然而, 对于稳定发光波 长、抑制外界刺激影响的研究却少有报道。压力作 为独立于温度和化学组分的热力学参量, 可以有效 地缩短原子间的距离, 使相邻轨道电子云重叠变大, 改变电子轨道行为和键合方式。ALIVISATOS 等 ${ }^{[13]}$ 早在 20 世纪 90 年代就率先开展了 $\mathrm{CdSe}$ 纳米晶的 高压光学响应研究, 并推进了后续的高压理论和实 验, 研究者已经在压力响应方面取得了新的研究成 果 ${ }^{[11-18]}$ 。例如, WANG 等 ${ }^{[16]}$ 研究发现: 高压下粒径 从 $5 \mathrm{~nm}$ 到 $1 \mu \mathrm{m}$ 的 $\mathrm{Y}_{2} \mathrm{O}_{3}$ 晶粒从晶态到非晶态转变 的尺寸依赖性，而体材料 $\mathrm{Y}_{2} \mathrm{O}_{3}$ 的晶体结构在压力 下却一直保持稳定, 没有发生非晶化现象。发生非 晶化现象与 $\mathrm{Y}_{2} \mathrm{O}_{3}$ 八面体长程有序在压力下被破坏有 关, 揭示了颗粒尺寸在高压下的变化。QUAN 等 ${ }^{[17]}$ 发现在 $8 \mathrm{GPa}$ 下, $13 \mathrm{~nm}$ 的 $\mathrm{PbTe}$ 纳米晶体发生了从 岩盐结构向中间相正交相的转变, 最终形成了 $\mathrm{CsCl}$ 结构; 而在同样的压力 $(8 \mathrm{GPa})$ 下, $5 \mathrm{~nm}$ 的 $\mathrm{PbTe}$ 纳米 晶体却转变为非晶态。卸压后 $13 \mathrm{~nm}$ 的 $\mathrm{PbTe}$ 纳米 晶结构可逆, $5 \mathrm{~nm}$ 的 $\mathrm{PbTe}$ 纳米晶却仍保持非晶状 态。 $\mathrm{PbTe}$ 在压力下的结构稳定性使其表现出反常霍 尔-佩奇效应, 这一现象为探索在实验室条件下形 成高压亚稳态结构和独特性能的新型材料提供了可 行途径。同时, 这些研究也为压力诱导的非晶态和 晶态之间的转换, 及生长过程的物理机制探索提供
了思路 ${ }^{[16-18]}$ 。然而, 对压力下光学抗压稳定性的研 究相对较少, 目前还未见有关压力对魔力尺寸材料 影响的研究。

本研究采用胶体化学方法合成 CdSe MSNs, 探 索 CdSe MSNs 在压力下的光学响应和稳定性, 为极 端压缩条件下的特殊魔力尺寸纳米材料的研究提供 参考。

\section{1 实验方法}

醋酸镉 $\left(\mathrm{Cd}(\mathrm{OAc})_{2} \cdot 2 \mathrm{H}_{2} \mathrm{O}, 99.5 \%\right)$ 、硒( $\left.\mathrm{Se}, 99.95 \%\right)$ 、 油酸 $(\mathrm{OA}, 90 \%)$ 和 1-十八烯(ODE，90\%)均购自阿拉 丁。甲苯、甲醇和丙酮为分析级, 购自北京化工公司。 实验中使用的所有化学制品均未进一步提纯。将 $0.2 \mathrm{mmol} \mathrm{Cd}(\mathrm{OAc})_{2} \cdot 2 \mathrm{H}_{2} \mathrm{O} 、 0.05 \mathrm{mmol} \mathrm{Se} 、 0.05 \mathrm{mmol}$ $\mathrm{OA}$ 和 $5 \mathrm{~g} \mathrm{ODE}$ 的混合物脱气 $30 \mathrm{~min}$, 升温到 $120{ }^{\circ} \mathrm{C}$ 维持 $1 \mathrm{~h}$ 。通过紫外-可见吸收光谱检测纳米晶的生 长过程。将合成的纳米晶分散在甲苯中, 滴到 300 目 $(45 \mu \mathrm{m})$ 的铜网上进行高分辨透射电子显微镜(HRTEM) 观察, 仪器型号为 JEM-2200FS, 加速电压为 $200 \mathrm{eV}$ 。

利用金刚石对顶砧压机进行高压实验，首先将 $\mathrm{T} 301$ 垫片预压至厚度 $45 \mu \mathrm{m}$, 然后在中心钻一个直 径为 $150 \mu \mathrm{m}$ 的孔。将 $\mathrm{CdSe}$ MSNs 装入样品腔中，同 时放入红宝石球标定压力, 由于红宝石的 $\mathrm{R} 1$ 和 R2 线的位置随着压力的升高近似线性移动, 因此可以 计算出腔内的压力值 $\left(P=\frac{1904}{7.665}\left[\left(\frac{\Delta \lambda}{\lambda_{0}}+1\right)^{7.665}-1\right]\right)$ 。 $\left(\lambda_{0}\right.$ 为红宝石发光的原始波长, $\Delta \lambda$ 为压力造成的红 宝石发光波长的变化)高压原位实验利用硅油作为 传压介质。利用半导体激光器作为激发光源(激发波 长为 $355 \mathrm{~nm}$ ), 激光功率为 $4.5 \mathrm{~mW}$, 积分时间为 $5 \mathrm{~s}$, 测试高压原位苂光光谱。以気卤灯为连续光源, 采用 海洋光学光纤光谱仪(型号 QE65000)测试高压原位 紫外可见吸收光谱。

\section{2 结果与讨论}

\section{1 常压下的结构和光学特性}

参照 $\mathrm{YU}$ 等 ${ }^{[2-3]}$ 提出的一锅煮液相合成法制备了 CdSe MSNs。图 1 为 CdSe MSNs 样品的吸收光谱、 发光光谱和晶体结构示意图。利用紫外-可见吸收光 谱监测 $\mathrm{CdSe}$ MSNs 的生长过程和电子结构的演变。 图 1(a)给出了不同条件合成样品的吸收光谱, 从图 中可以看出样品呈现两个尖锐的吸收峰, 这两个光 学吸收峰与不同的电子跃迁有关, 分别对应于从 

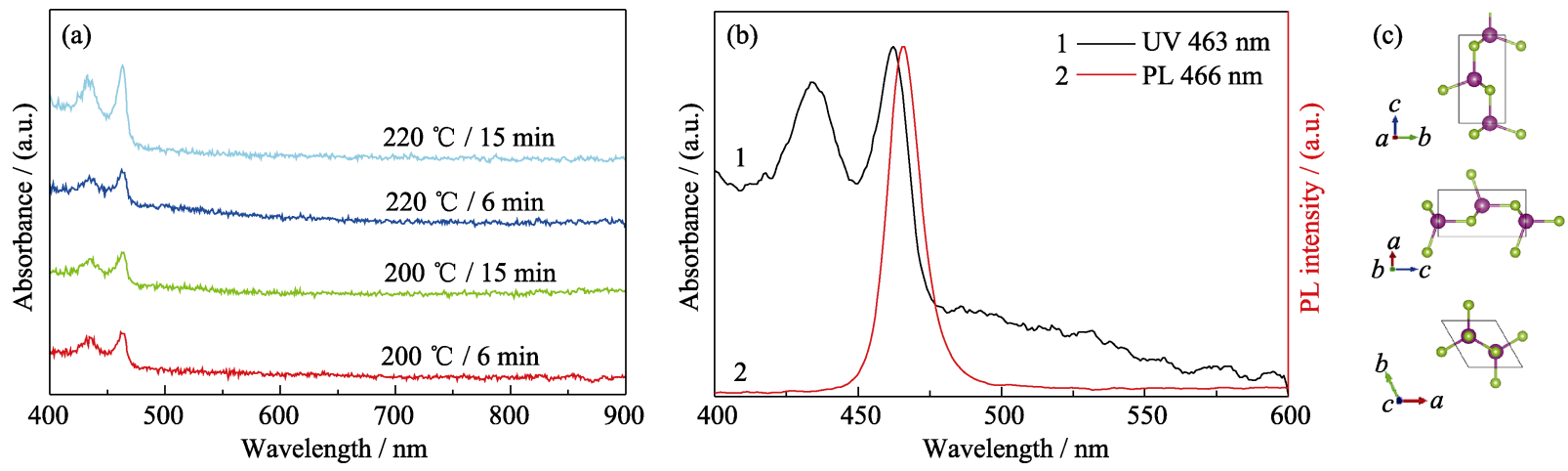

图 1 (a)分散在甲苯中的 CdSe MSNs 随时间和温度演化的吸收光谱; (b)200 ${ }^{\circ} \mathrm{C} / 6 \mathrm{~min}$ 制备样品的归一化吸收和发光光谱 (激发波长 $355 \mathrm{~nm}$ ); (c) $a, b, c$ 方向上纤锌矿 $\mathrm{CdSe}$ 的晶体结构示意图

Fig. 1 (a) Absorption spectra of time-temperature evolution of CdSe MSNs samples dispersed in toluene, (b) normalized absorption spectrum (black line, left $y$-axis) and emission (red line, excitation wavelength $355 \mathrm{~nm}$, right $y$-axis) of CdSe MSNs obtained at $200{ }^{\circ} \mathrm{C} / 6 \mathrm{~min}$, and (c) schematic of crystal structure of CdSe in wurtzite (from three different directions of $a, b$ and $c$, respectively)

价带的轻空穴和重空穴能级到导带的最低能级的跃

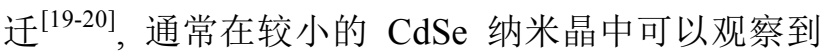

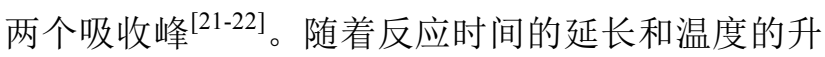
高, 样品的第一激子吸收峰逐渐增强, 在 $463 \mathrm{~nm}$ 处 的吸收峰位不变, 表明合成的样品发生了不同于常 规量子点的异质生长。同时, 样品的第一激子吸收峰 的半高宽非常小, 仅为 $13 \mathrm{~nm}$, 远小于 $\mathrm{CdSe}$ 常规量 子点的半高宽, 进一步表明所制备的样品是 $\mathrm{MSNs}^{[1]}$ 。

图 1(b)为温度 $200{ }^{\circ} \mathrm{C}$ 、反应时间 $6 \mathrm{~min}$ 获得的 CdSe MSNs 归一化的吸收光谱和发光光谱。CdSe MSNs 的稳定性抑制了纳米颗粒的生长 ${ }^{[8-9,23]}$, 相对 较窄的光吸收表明合成的 CdSe MSNs 具有相对均 匀的尺寸分布。由于存在表面缺陷，抑制了 MSNs 的发光。此外, 具有高能量的带边也使表面原子的 钝化变得困难, 从而难以捕获光激发载流子。在已 有的 CdSe MSNs 研究报道中, 这些纳米晶体表现为 可见光范围内宽带发射 $(420 \sim 710 \mathrm{~nm})$, 不受自身吸 收的影响。CdSe MSNs 的宽带发射归因于隙间表面

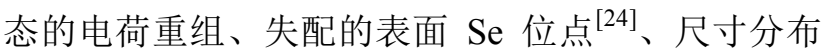
窄以及斯托克斯位移 $(40 \sim 50 \mathrm{~nm})$ 大。通过一锅煮法 合成的 CdSe MSNs 通过电子和空穴直接复合具有 窄发射特征。由于表面配体能与纳米晶体表面的金 属原子结合形成配位键, 因此, 通过表面配体调节 纳米晶的表面自由能, 稳定或破坏特定晶面, 从而 影响胶体纳米晶的成核和生长动力学。在 $\mathrm{CdSe}$ MSNs 的合成过程中, 表面配体决定其生长速率及 发光特性。在反应系统中添加长链脂肪酸时, 脂肪 酸充当表面配体, 将 $\mathrm{CdSe}$ MSNs 表面与乙酸基团一 起钝化, 表面上的长链脂肪酸抑制了纳米颗粒的聚 集 ${ }^{[25]}$ 。Kudera 等 ${ }^{[1]}$ 采用饱和的壬酸作为表面配体, 合成的 CdSe MSNs 带边吸收位于 $446 \mathrm{~nm}$, 处于连 续生长状态。本研究用链更长的不饱和脂肪酸油酸
作为表面配体, 发现在 $463 \mathrm{~nm}$ 处存在稳定的 MSNs, 并具有带边吸收和发光, 原因在于长链不饱和的油 酸配体能够通过与纳米晶表面结合, 允许 $\mathrm{CdSe}$ MSNs 生长 ${ }^{[2-3]}$ 。

此外，非共振斯托克斯位移(Non-Resonant Stokes Shift, NRSS)是带边吸收和发光峰位之间的能量差, 它受精细结构能级间距、纳米晶内弛豫过程和纳米 晶尺寸分布的影响 ${ }^{[26]}$, 图 1(b)中制备的 CdSe MSNs 的 NRSS 只有 $186 \mathrm{meV}$ 。超小的 NRSS 以及稳定的 生长动力学, 可以制备高质量的 CdSe MSNs, 它具 有窄的发射带宽和高的发光强度 ${ }^{[2,8-9]}$ 。图 1(c)给出 了沿着 $a 、 b 、 c$ 三个方向上呈现的纤锌矿 $\mathrm{CdSe}$ 晶 体结构示意图，属于六角晶系, Se 原子呈六方密堆 积, $\mathrm{Cd}$ 原子填充在四面体空隙中, 配位数均为 4 。

图 2 为 $\mathrm{CdSe}$ MSNs 的 HRTEM 照片, 晶格条纹

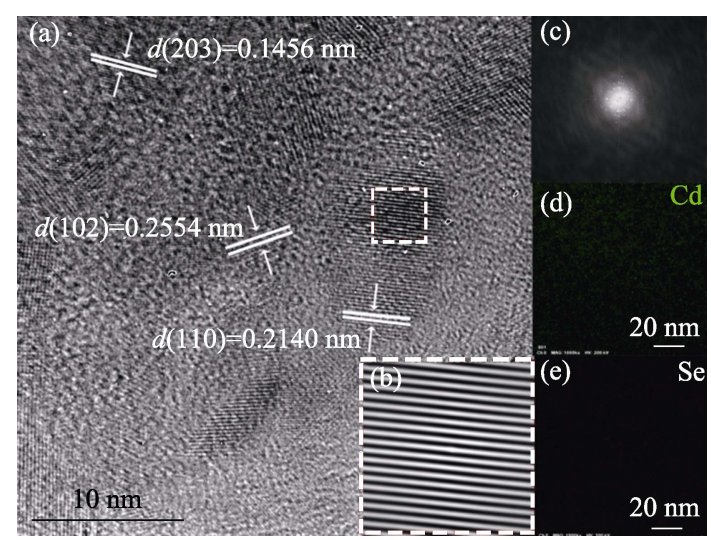

图 2 (a)CdSe MSNs 的高分辨透射电子显微镜照片; (b)图(a) 中对应区域的原子有序排列视图; (c)CdSe MSNs 的选区电子 衍射图像; (d, e) 为 CdSe MSNs 不同元素的面分布

Fig. 2 (a) HRTEM image of CdSe MSNs with (b) corresponding selected area correlation pattern of the square in (a), (c) FFTHRTEM image of CdSe MSNs, and (d, e) elemental mapping images of CdSe MSNs 
清晰, 晶格间距 $(d)$ 为 $0.1456 、 0.2140$ 和 $0.2554 \mathrm{~nm}$, 分别对应于六方晶系 CdSe 的(203)、(110)和(102)晶 面。点线选区 (b)为原子有序排列视图。通过对 HRTEM 照片进行快速傅里叶变换(FTT), 可以得到 同心圆的电子衍射花样(图 2(c)), 表明 CdSe MSNs 具有多个晶面的多晶结构。图 2(d,e)为 CdSe MSNs 的元素面分布图像。由于纳米晶体的尺寸较小, 其 分布较为分散且均匀。

\section{2 压力下的光学特性表征}

在 MSNs 的基础上引入压力维度, 探索高压下 的结构和光学稳定性。采用金刚石对顶砧技术对 CdSe MSNs 进行压力原位测试。图 3(a)显示了随着

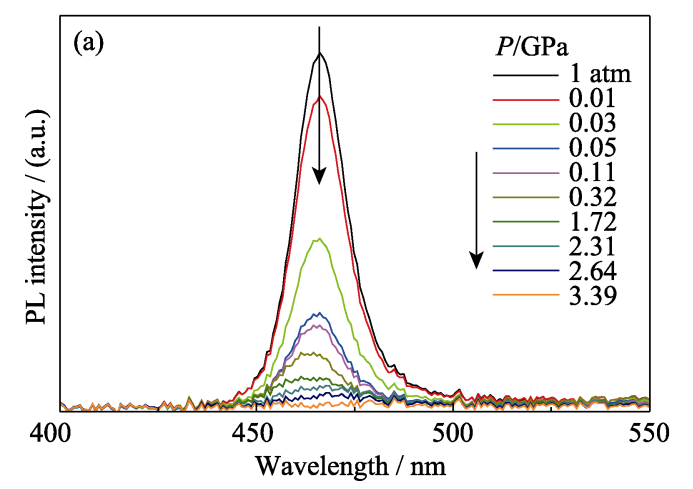

压力的增大, 苂光强度逐渐衰减; 当压力增加到 $3.39 \mathrm{GPa}$ 时, 荧光消失。值得注意的是, 在压力作用 下, CdSe MSNs 的苂光峰位置保持不变, 发光峰位 对外界压力刺激具有很好的稳定性。同时, 卸压后 荧光峰位置图 3(b)也没有发生变化, 因此合成的 CdSe MSNs 具有类似于 Richle 等 ${ }^{[27]}$ 报道的魔力尺寸 $(\mathrm{CdSe})_{n}(n=33,34)$ 分子核笼结构, 较高波长处的第 一激子吸收峰表明原子数 $n$ 较高, 带间的荧光与团 簇的对称性相关。上述研究表明, 当材料的尺寸小 到团簇大小时, 压力变化对其团簇内部对称性无影 响，使其在极端压缩条件下的发光峰位不变。

图 4 为 $\mathrm{CdSe}$ MSNs 在高压下的原位紫外-可见

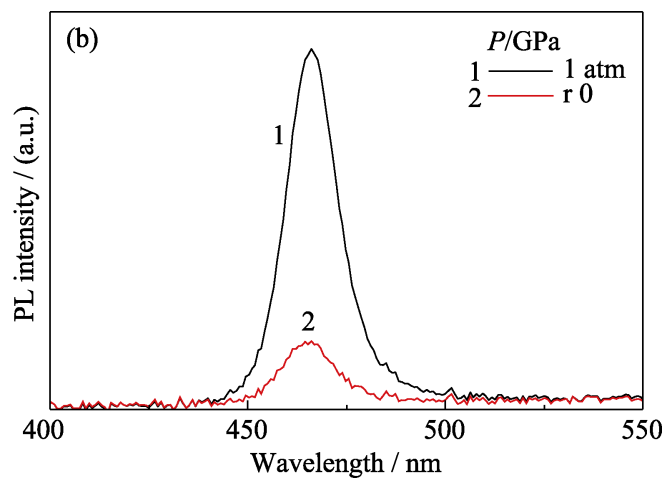

图 3 (a)在不同压力下 CdSe MSNs 的苂光光谱, (b)卸压后 CdSe MSNs 的发光光谱与常压发光光谱的比较 $(1 \mathrm{~atm}=101325 \mathrm{~Pa})$

Fig. 3 (a) High pressure photoluminescence spectra of CdSe MSNs, and (b) comparison of photoluminescence spectra of CdSe MSNs before and after compression (1 atm=101325 Pa)

$\mathrm{r} 0$ dipicts the ambient pressure after completely releasing pressure
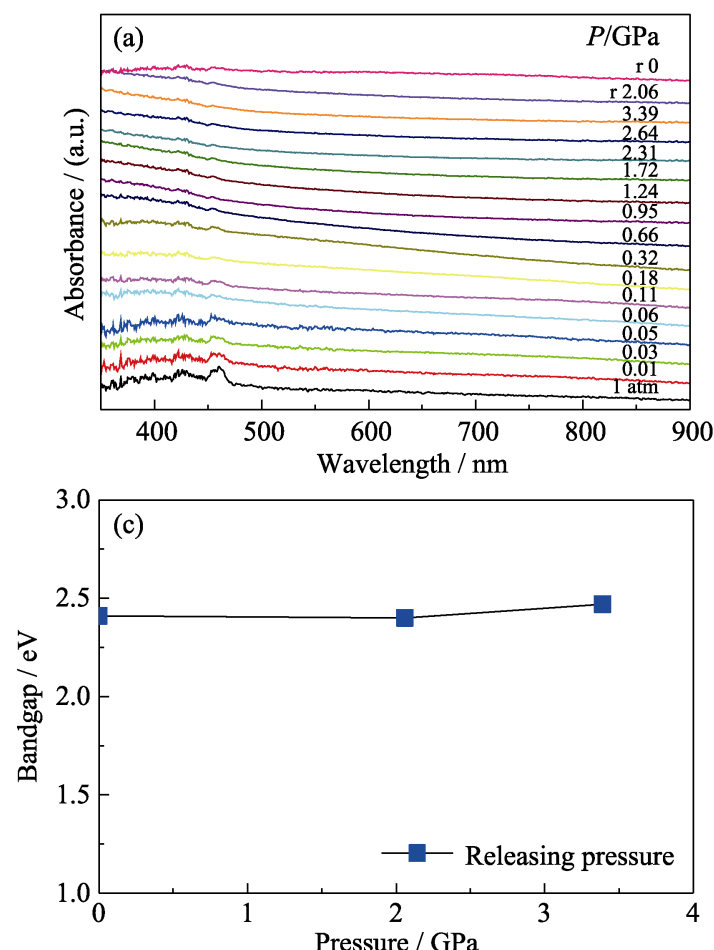

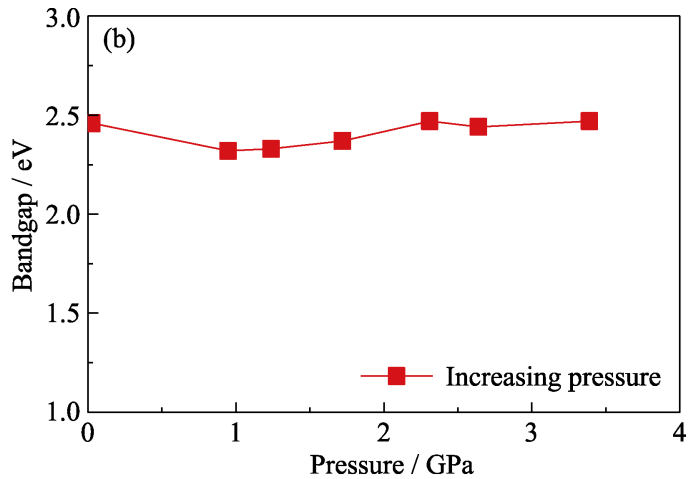

(d)

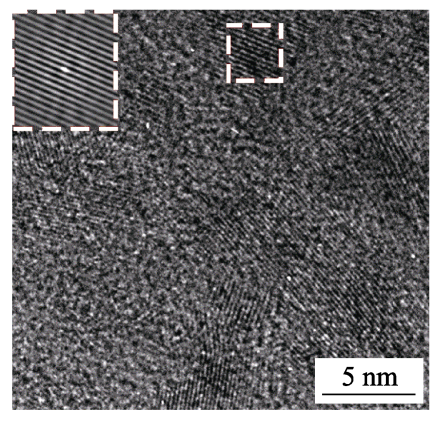

图 $4 \mathrm{CdSe}$ MSNs 在高压下的原位紫外-可见吸收光谱(a), 加压和卸压过程中的带隙演化(b, c), 卸压后的 HRTEM 照片(d)

Fig. 4 (a) In situ high pressure UV-Vis absorption spectra, (b, c) band gap evolution of CdSe MSNs in the process of pressurization and pressure relief, and (d) HRTEM image of CdSe MSNs after the pressure relief $\mathrm{r} 2.06$ and $\mathrm{r} 0$ in (a) represent the pressure released to $2.06 \mathrm{GPa}$ and ambient conditions 
吸收光谱, 从图 4(a)中可以看出, CdSe MSNs 的吸 收双峰位置在压力作用下未发生移动。由于吸收峰 位的变化与带隙的变化直接相关, 因此, 通过不同 压力下的紫外-可见吸收光谱, 可以得到高压下 CdSe MSNs 的带隙演化。图 4(b,c) 分别为在加压和 卸压过程中 CdSe MSNs 带隙的变化曲线, 可以看出, 随着压力的增大或减小, CdSe MSNs 的带隙基本保 持不变, 带隙随压力的变化可以近似看作是一条水 平直线; 卸压后还可以清晰地看到 $\mathrm{CdSe}$ MSNs 的晶 格(图 4(d))。魔力尺寸 $\mathrm{CdSe}$ 纳米晶的特殊结构、单 一尺寸和精确原子配比使其具有良好的压力稳定性, 光物理性能对外界压力无响应。

\section{3 结论}

利用金刚石对顶砧压机对 CdSe MSNs 进行了 高压原位苂光和高压原位吸收光谱表征, 发现 $\mathrm{CdSe}$ MSNs 具有良好的耐压性能。与传统的半导体纳米 材料在高压下所发生的峰位红移或蓝移完全不同, $\mathrm{CdSe} \mathrm{MSNs}$ 的荧光强度在压力作用下衰减的同时, 发光峰位置保持不变; 卸压后, 荧光峰位置也没有 发生变化。CdSe MSNs 的带隙同样在压力作用下保 持稳定。

\section{参考文献:}

[1] KUDERA S, ZANELLA M, GIANNINI C, et al. Sequential growth of magic-size CdSe nanocrystals. Advanced Materials, 2007, 19(4): $548-552$.

[2] YU K, OUYANG J Y, ZAMAN M B, et al. Single-sized CdSe nanocrystals with bandgap photoemission via a noninjection one-pot approach. The Journal of Physical Chemistry C, 2009, 113(9): 3390-3401.

[3] OUYANG J Y, ZAMAN M B, YAN F J, et al. Multiple families of magic-sized CdSe nanocrystals with strong bandgap photoluminescence via noninjection one-pot syntheses. The Journal of Physical Chemistry C, 2008, 112(36): 13805-13811.

[4] BOWERS M J, MCBRIDE J R, ROSENTHAl S J. White-light emission from magic-sized cadmium selenide nanocrystals. Journal of the American Chemical Society, 2005, 127(44): 15378-15379.

[5] DAGTEPE P, CHIKAN V, JASINSKI J, et al. Quantized growth of CdTe quantum dots; observation of magic-sized CdTe quantum dots. The Journal of Physical Chemistry C, 2007, 111(41): 14977-14983.

[6] DUKES A D, MCBRIDE J R, ROSENTHAL S J. Synthesis of magic-sized CdSe and CdTe nanocrystals with diisooctylphosphinic acid. Chemistry of Materials, 2010, 22(23): 6402-6408.

[7] LI M, OUYANG J, RATCIFFE C I, et al. CdS magic-sized nanocrystals exhibiting bright band gap photoemission via thermodynamically driven formation. ACS Nano, 2009, 3(12): 3832-3838.

[8] GAO D, HAO X Y, ROWELL N, et al. Formation of colloidal alloy semiconductor $\mathrm{CdTeSe}$ magic-size clusters at room temperature. Nature Communications, 2019, 10(1): 1674.

[9] YANG J W, MUCKEL F, BAEK W, et al. Chemical synthesis, doping, and transformation of magic-sized semiconductor alloy nanoclusters. Journal of the American Chemical Society, 2017, 139(19): 6761-6770.

[10] CHEN M, LUAN C R, ZHANG M, et al. Evolution of CdTe magic-size clusters with single absorption The Journal of Physical Chemistry Letters, 2020, 11(6): 2230-2240.

[11] XIAO G J, WANG Y N, HAN D, et al. Pressure-induced large emission enhancements of cadmium selenide nanocrystals. Journal of the American Chemical Society, 2018, 140(42): 13970-13975.

[12] LV P F, YANG S R, LIU C, et al. Pressure-induced emission enhancements and ripening of zinc blende cadmium selenide nanocrystals. The Journal of Physical Chemistry C, 2019, 123(24): 15339-15344.

[13] TOLBERT S H, ALIVISATOS A P. The wurtzite to rock salt structural transformation in CdSe nanocrystals under high pressure. The Journal of Chemical Physics, 1995, 102(11): 4642-4656.

[14] SHI Y, MA Z W, ZHAO D L, et al. Pressure-induced emission (PIE) of one-dimensional organic tin bromide perovskites. Journal of the American Chemical Society, 2019, 141(16): 6504-6508.

[15] MA Z W, LIU Z, LU S Y, et al. Pressure-induced emission of cesium lead halide perovskite nanocrystals. Nature Communications, 2018, 9(1): 4506.

[16] WANG L, YANG W G, DING Y, et al. Size-dependent amorphization of nanoscale $\mathrm{Y}_{2} \mathrm{O}_{3}$ at high pressure. Phys. Rev. Lett., 2010, 105(9): 095701.

[17] QUAN Z W, WANG Y X, BAE I T, et al. Reversal of Hall-Petch effect in structural stability of PbTe nanocrystals and associated variation of phase transformation. Nano Letters, 2011, 11(12): 5531-5536.

[18] QUAN Z W, LUO Z P, WANG Y X, et al. Pressure-induced switching between amorphization and crystallization in PbTe nanoparticles. Nano Letters, 2013, 13(8): 3729-3735.

[19] LYASHCHOVA A, DMYTRUK A, DMITRUK I, et al. Optical absorption, induced bleaching, and photoluminescence of CdSe nanoplatelets grown in cadmium octanoate matrix. Nanoscale Research Letters, 2014, 9(1): 88.

[20] NIRMAL M, NORRIS D J, KUNO M, et al. Observation of the “dark exciton" in CdSe quantum dots. Physical Review Letter, 1995, 75(20): 3728-3731.

[21] NORRIS D J, EFROS A L, ROSEN M. Size dependence of exciton fine structure in CdSe quantum dots. Physical Review B, 1996, 53(24): $16347-16354$.

[22] NORRIS D J, BAWENDI M G. Measurement and assignment of the size-dependent optical spectrum in CdSe quantum dots. Physical Review B, 1996, 53(24): 16338-16346.

[23] NEVERS D R, WILLIAMSON C B, SAVITZKY B H, et al. Mesophase formation stabilizes high-purity magic-sized clusters. Journal of the American Chemical Society, 2018, 140(10): 3652-3662.

[24] GROENEVELD E, VAN BERKUM S, MEIJERINK A, et al. Growth and stability of ZnTe magic-size nanocrystals. Small, 2011, 7(9): 1247-1256.

[25] LIU X M, JIANG Y, WANG C, et al. White-light-emitting CdSe quantum dots with "magic size" via one-pot synthesis approach. Physica Status Solidi (A), 2010, 207(11): 2472-2477.

[26] EFROS A L, ROSEN M, KUNO M, et al. Band-edge exciton in quantum dots of semiconductors with a degenerate valence band: dark and bright exciton states. Physical Review B, 1996, 54(7): 4843-4856.

[27] RIEHLE F S, BIENERT R, THOMANN R, et al. Blue luminescence and superstructures from magic size clusters of CdSe. Nano Letters, 2009, 9(2): 514-518. 